\title{
Frequency-Comb-Assisted Terahertz Quantum Cascade Laser Spectroscopy
}

\author{
S. Bartalini, ${ }^{*}$ L. Consolino, P. Cancio, and P. De Natale \\ INO, Istituto Nazionale di Ottica-CNR, Largo Fermi 6, 50125 Firenze, Italy \\ and LENS, European Laboratory for Non-linear Spectroscopy, \\ Via Carrara 1, 50019 Sesto Fiorentino, Italy \\ P. Bartolini, A. Taschin, and M. De Pas \\ LENS, European Laboratory for Non-linear Spectroscopy, Via Carrara 1, 50019 Sesto Fiorentino, Italy \\ H. Beere and D. Ritchie \\ Cavendish Laboratory, University of Cambridge, J. J. Thomson Avenue, \\ Cambridge CB3 OHE, United Kingdom \\ M. S. Vitiello \\ INO, Istituto Nazionale di Ottica-CNR, Largo Fermi 6, 50125 Firenze, Italy \\ and NEST, Istituto Nanoscienze-CNR and Scuola Normale Superiore, \\ Piazza San Silvestro 12, 56127 Pisa, Italy \\ R. Torre \\ LENS, European Laboratory for Non-linear Spectroscopy, Via Carrara 1, 50019 Sesto Fiorentino, Italy \\ and Dipartimento di Fisica e Astronomia, Università di Firenze, \\ Via Sansone 1, 50019 Sesto Fiorentino, Italy \\ (Received 23 October 2013; revised manuscript received 23 December 2013; published 9 April 2014) \\ We report a metrological-grade THz spectroscopy based on the combination of a THz frequency-comb \\ synthesizer (FCS) and a THz quantum cascade laser (QCL). The QCL, emitting at $2.5 \mathrm{THz}$, is phase locked \\ to the free-space THz FCS, and its frequency is swept across a methanol transition by tuning the comb- \\ repetition rate, which is ultimately disciplined by the Cs primary frequency standard. The absolute \\ frequency scale provides an uncertainty of a few parts in $10^{-11}$ on the laser frequency and $10^{-9}$ on the line- \\ center determination, ranking this technique among the most precise ever developed in the $\mathrm{THz}$ range.
}

DOI: 10.1103/PhysRevX.4.021006

\section{INTRODUCTION}

Terahertz quantum cascade lasers (QCLs) [1] are proving to be the key technology for countless applications [2-6], recently unveiling a quantum-limited spectral purity below any other semiconductor source $[7,8]$. In parallel, the very recent extension of frequency-comb synthesizers (FCSs) to the far infrared enables direct and broadband phase or frequency referencing for any source in this range [9-11], providing a new tool for high-resolution measurements of $\mathrm{THz}$ frequencies. The interest in metrological-grade $\mathrm{THz}$ molecular spectroscopy has deep motivations. In this spectral range, rotational transitions of simple molecules have Doppler-limited and natural linewidths that can be as

\footnotetext{
*saverio.bartalini@ino.it
}

Published by the American Physical Society under the terms of the Creative Commons Attribution 3.0 License. Further distribution of this work must maintain attribution to the author $(s)$ and the published article's title, journal citation, and DOI.
Subject Areas: Atomic and Molecular Physics, Photonics

narrow as a few hundred $\mathrm{kHz}$ and a few $\mathrm{Hz}$, respectively. As a consequence, spectroscopy on low-pressure and/or cold-gas samples can only be challenged by narrowemission sources that have a precisely controlled frequency. For almost two decades, since their first demonstration [12], tunable far-infrared (TuFIR) sources have been employed for a variety of challenging high-precision experiments [13-16]. Despite their inherent limitations, such as the very low power (tens of nW) and the bulky and complex instrumentation, TuFIR sources have been, for a long time, the only tool for measuring the frequency of $\mathrm{THz}$ transitions with a $10^{-8}$ accuracy. As an alternative, frequency-multiplied microwave sources have recently been extended to the $\mathrm{THz}$ region [17], providing $\mu \mathrm{W}$-power radiation up to $2.8 \mathrm{THz}$, with a tuning range of about $10 \%$ around the central emission frequency and an accuracy of $10^{-8}$ on the center of the transition frequency. At the same time, promising approaches to high-resolution $\mathrm{THz}$ spectroscopy inspired by the TuFIR systems have recently been developed; they are based on difference-frequency generation of $\mathrm{THz}$ light by mixing visible or near-infrared lasers 
$[18,19]$. All these techniques, although inherently different, have a common feature, i.e., the indirect generation of $\mathrm{THz}$ radiation, produced via frequency mixing of sources emitting in other spectral ranges. If, on one hand, frequencymixing processes allow, in principle, an easy referencing of the $\mathrm{THz}$ radiation to primary or secondary frequency standards, on the other hand they make use of lowefficiency frequency-conversion mechanisms, resulting in $\mathrm{THz}$ radiation powers typically below a few $\mu \mathrm{W}$. Low power can be the main limiting factor for a variety of highresolution spectroscopic techniques, such as sub-Doppler spectroscopy, that are indeed crucial for a full exploitation of metrological-grade $\mathrm{THz}$ sources. In this perspective, the development of relatively high-power, continuous-wave $\mathrm{THz}$ QCL sources offers the opportunity to extend across the $\mathrm{THz}$ gap state-of-the-art spectroscopic techniques that were developed in other spectral regions. QCLs are, indeed, ideal candidates for $\mathrm{THz}$ metrology, since they have shown an inherently high spectral purity, with intrinsic linewidths as low as $100 \mathrm{~Hz}$ [7]. However, their typical free-running linewidths are in the range of $1 \mathrm{MHz}$, due to the presence of excess frequency noise that must be removed in order to exploit their metrological-grade potential. A THz FCS, disciplined by a primary standard, can be a suitable narrow and stable frequency reference, providing not only a reduction of QCLs frequency fluctuations down to the quantum limit but also control on their absolute emission frequency. Only a few techniques have been reported, so far, for linking a THz QCL to mode-locked near-IR lasers [9-11]. They all provide a comblike metrological reference for phase locking any continuous-wave source over a wide spectral window, ranging from the $\mathrm{GHz}$ range up to several THz. The approach followed by our group, based on an airpropagating $\mathrm{THz}$ radiation $\mathrm{FCS}$, allows us to minimize the power of the THz QCL needed for the phase lock, thus leaving most of the power for further spectroscopic (or other) applications [11].

\section{EXPERIMENT}

The experiment aims to give a proof of principle of the all-optical approach to $\mathrm{THz}$ metrology, and, for this reason, the experimental scheme has been kept as simple as possible (see Fig. 1). The QCL employed for the experiment is based on a GaAs/ $\mathrm{Al}_{0.15} \mathrm{Ga}_{0.85} \mathrm{As}$ heterostructure and on a standard surface-plasmon waveguide design, and emits at $2.5 \mathrm{THz}$. It is housed on the cold finger of a liquid$\mathrm{He}$ cryostat driven in continuous-wave mode at a fixed-heat sink temperature $T_{o} \sim 47.5 \mathrm{~K}$. Under these experimental conditions, the QCL threshold current is $I_{\text {th }}=375 \mathrm{~mA}$, and the output power corresponding to the operating current $I_{o}=430 \mathrm{~mA}$ is $P_{\text {out }} \sim 1 \mathrm{~mW}$. A direct-absorption spectroscopy setup has been implemented on a 10-cm-long cell filled with methanol gas, using the available fraction of the QCL beam (more than 99\% of the total power) and a room-temperature pyroelectric detector, together with an optical chopper on the beam and a and lock-in acquisition. The remaining small fraction of the QCL power is used for the phase lock to the $\mathrm{THz}$ comb, following the same scheme described in Ref. [11].

The mode-locked Ti:sapphire laser used for $\mathrm{THz}$ comb generation is actively stabilized against a $10-\mathrm{MHz}$ quartz-oscillator disciplined by a Rb-GPS (Global Positioning System) clock (stability of $6 \times 10^{-13}$ in $1 \mathrm{~s}$ and absolute accuracy of $2 \times 10^{-12}$ ). In this way, we transfer the traceability of the Cs primary frequency standard to the Ti:sapphire repetition rate $\left(f_{\text {rep }}=\right.$ $77.5 \mathrm{MHz}$ ). The measured $f_{\text {rep }}$ Allan variance is about $4 \mathrm{mHz}$ at $1 \mathrm{~s}$ and $1 \mathrm{mHz}$ at $100 \mathrm{~s}$, corresponding to a relative stability always better than $5 \times 10^{-11}$. Given the generation mechanism of the zero-offset $\mathrm{THz}$ FCS that can be interpreted as a difference-frequency generation between teeth pairs of the pump laser, the frequency of each comb tooth is $N$ times the $f_{\text {rep }}, N$ being the order of the tooth. As a consequence, any common-mode (offset-type) instability of the fs laser is not propagated to the teeth of the $\mathrm{THz}$ comb. The stability of each tooth is therefore given by the product of the measured stability of $f_{\text {rep }}$ and the tooth order $N$. At $2.5 \mathrm{THz}$, with an order $N \sim 32950$, we obtain a stability of about $130 \mathrm{~Hz}$. Regarding the linewidth of the $\mathrm{THz}$ comb tooth, a direct measurement performed at $100 \mathrm{GHz}$ on a similar $\mathrm{THz}$ comb by heterodyne beating with a frequency-multiplied source [20] suggests, if the proper scaling with frequency is taken into account, a value of a few hundreds of Hz. Thanks to the phase lock with the $\mathrm{THz}$ comb, about $75 \%$ of the optical power of our $\mathrm{THz}$ QCL is narrowed down to the comb-tooth linewidth, thus approaching its intrinsic linewidth and providing a $5 \times 10^{-11}$ uncertainty in the determination of its absolute frequency. The beat-note frequency $f_{b}$ is kept constant by the phase-lock loop, as described in detail in Ref. [11]. As a consequence, the QCL absolute frequency can be retrieved by

$$
\nu_{\mathrm{QCL}}=N f_{\text {rep }} \pm f_{b},
$$

once the order $N$ of the beating tooth is known (see below). Moreover, the tuning of the QCL absolute frequency can be achieved by controlling the Ti:sapphire $f_{\text {rep }}$, since the QCL frequency rigidly follows the sweeping tooth of the $\mathrm{THz}$ comb (Fig. 1). An example is given in Fig. 2, where the spectroscopic signal acquired while tuning the QCL across a pair of methanol lines is shown. The 170-MHz-wide frequency scan is achieved by tuning $f_{\text {rep }}$ of about $5 \mathrm{kHz}$, with a 1-Hz-step linear ramp (about 33-kHz steps for the QCL frequency). The identification of these lines, however, depends on the knowledge of the QCL absolute frequency, that, in turn, depends on the identification of the tooth $N$ involved in the beating process, as described by Eq. (1), where the sign of $f_{b}$ depends on whether the QCL frequency is higher or lower than the tooth one. 


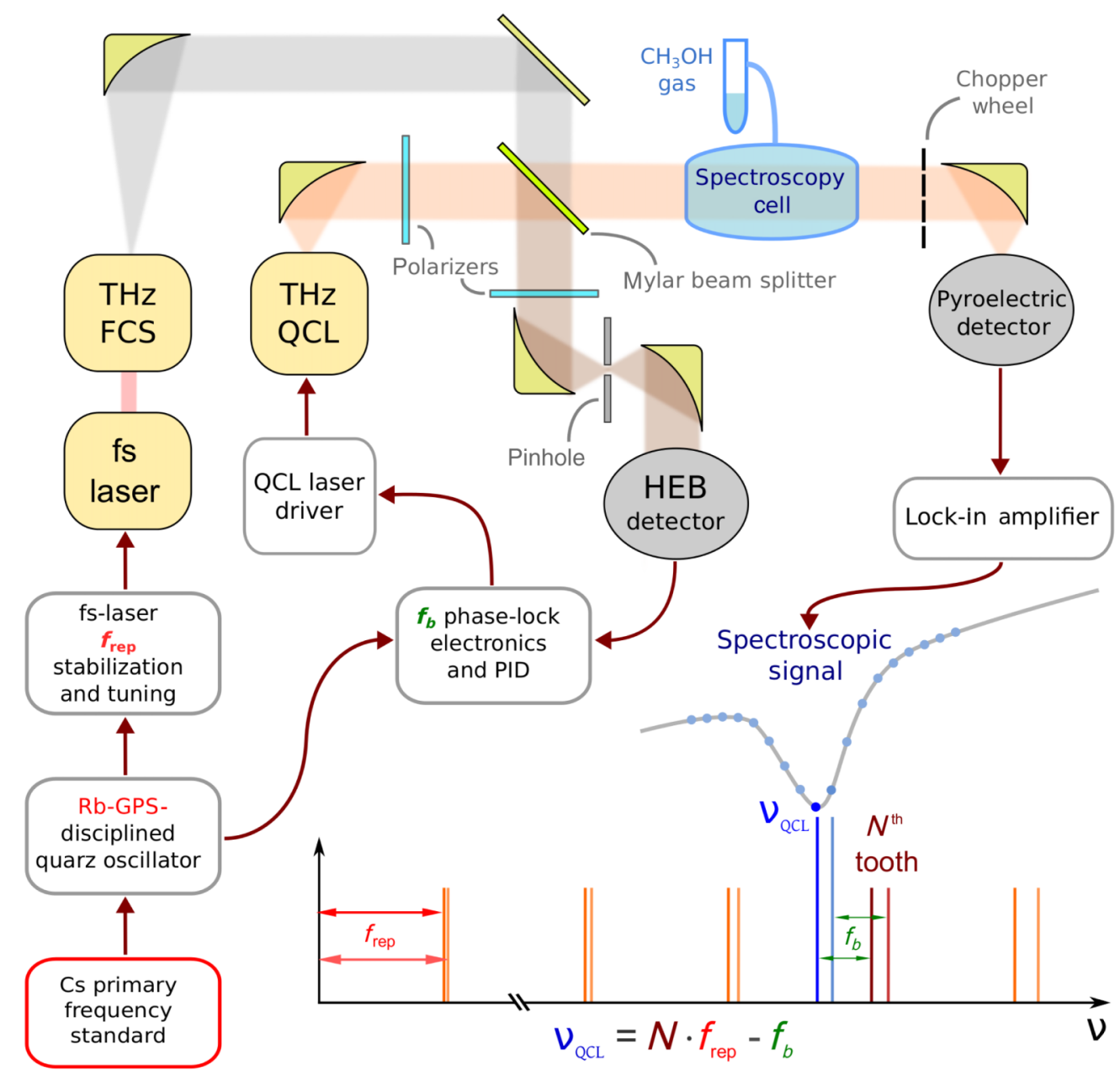

FIG. 1. The scheme of the experimental setup is shown, with the diagram describing how the traceability of the primary Cs frequency standard is transferred to the THz QCL-based spectroscopy via the stabilization of the repetition rate $\left(f_{\text {rep }}\right)$ of the pump laser and the THz FCS. The mechanism for tuning the QCL frequency $\left(\nu_{\mathrm{QCL}}\right)$ is also sketched: The beat-note frequency $\left(f_{b}\right)$ is kept constant by the phaselock loop, while the tuning of $f_{\text {rep }}$ produces a proportional shift of the THz comb tooth, and thus of the QCL absolute frequency. The lock-in acquisition of the absorption signal requires the beam intensity to be mechanically modulated by a chopper. HEB-hot-electron bolometer; PID-proportional-intergal-derivative controller.

An independent measurement of the THz QCL frequency with an accuracy better than $f_{\text {rep }}$ is not, presently, achievable by any commercial instrument (such as a FTIR spectrometer). Moreover, the narrow tuning range of the QCL prevents the acquisition of a larger number of consecutive methanol lines, making difficult their direct identification on a molecular database. For this reason, we need a preliminary estimation of $N$ in order to select a narrower spectral window in which to interrogate the molecular database. Among the few techniques demonstrated in other spectral regions for determining $N$ [21,22], we adopt an approach that has also been used in the sub$\mathrm{THz}$ domain [23]. Basing it on Eq. (1), it consists in tuning the $\mathrm{THz}$ comb-repetition rate by a certain amount $\left(\Delta f_{\text {rep }} \sim 50 \mathrm{kHz}\right.$, in our case) and measuring the consequent variation of the frequency of the beat note with a specific tooth $\left(\Delta f_{b} \sim 1.6 \mathrm{GHz}\right)$. The tooth order $N$ is then retrieved by

$$
N=\mp \frac{\Delta f_{b}}{\Delta f_{\text {rep }}}
$$

During the measurements, the QCL frequency must be kept constant. In our case, it has been stabilized against the side of the methanol transition by implementing a software proportional-integral loop on the QCL current and using the absorption profile as a feedback signal. From the above procedure, a value $N=32930 \pm 66$ is retrieved. The error, described by

$$
\delta N=N\left|\frac{\delta\left(\Delta f_{b}\right)}{\Delta f_{b}}\right|+N\left|\frac{\delta\left(\Delta f_{\text {rep }}\right)}{\Delta f_{\text {rep }}}\right|
$$

is dominated by the large relative uncertainty on $\Delta f_{b}$ (about $0.2 \%$ ) due to the performances of the QCL frequency-stabilization loop, while the relative uncertainty on $\Delta f_{\text {rep }}$ is negligible. This result, alone, does not allow us to 


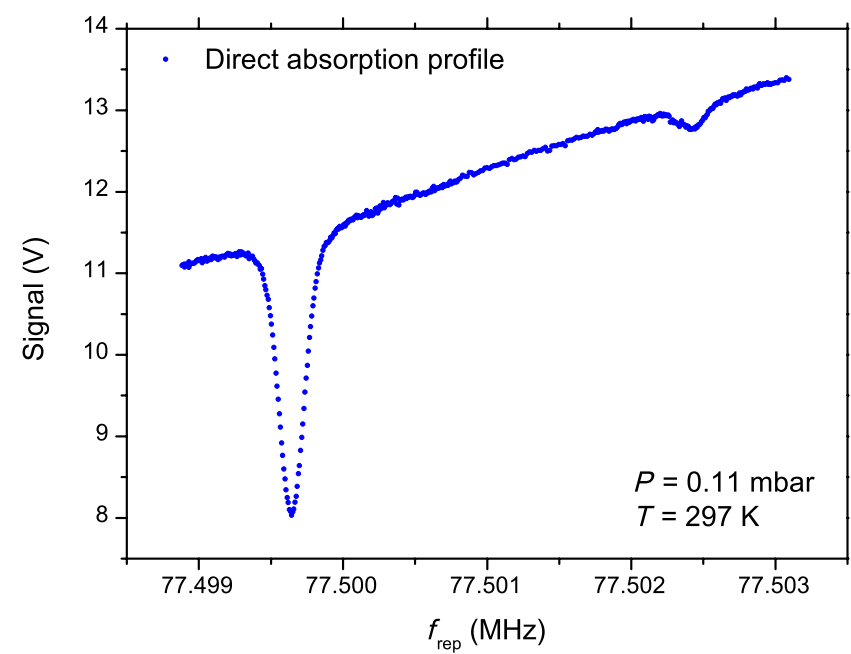

FIG. 2. Spectroscopic signal, recorded by tuning the repetition rate of the Ti:sapphire pump laser. Experimental conditions (gas pressure and temperature) are also reported.

univocally determine either $N$ or the QCL absolute frequency. Nevertheless, it allows us to narrow the search of the detected molecular transition within a $10-\mathrm{GHz}$-wide spectral window, where only a few tens of methanol lines are present (Fig. 3). Among these lines (as listed by the Molecular Spectroscopy Database of CalTech JPL [25]), there is only one whose frequency is consistent with Eq. (1), calculated with the experimental values of $f_{\text {rep }}$

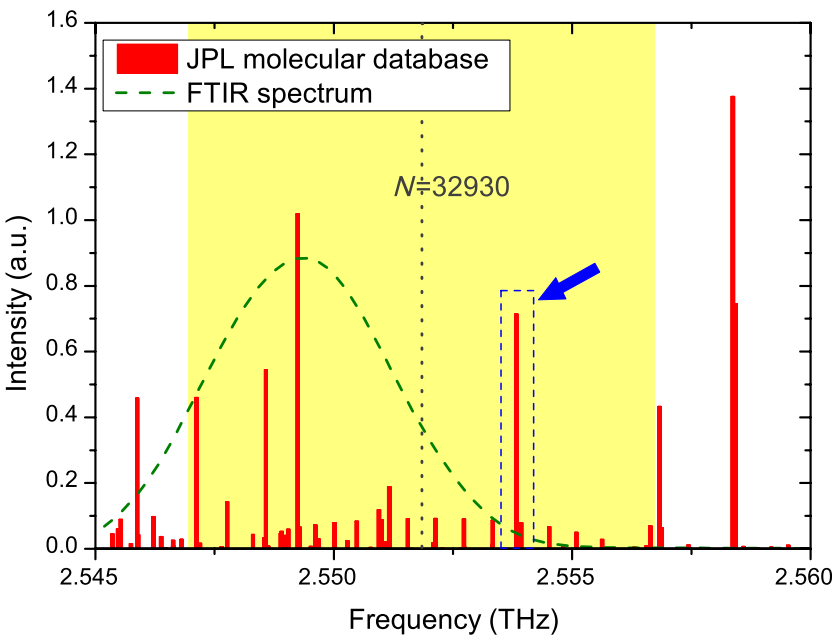

FIG. 3. The procedure for the determination of $N$ selects a group of a few tens of lines (within a $10-\mathrm{GHz}$-wide windowlight yellow region) where one may look for the detected line. This line is the only one (blue arrow) whose listed frequency can be retrieved, at the sub-MHz level, by Eq. (1) with the experimental $f_{\text {rep }}$ and $f_{b}$ values and an integer value of $N$ (see the Supplemental Material [24]). The presence of the pair provides a further confirmation. It is worth noting that a preliminary measurement of the QCL frequency, provided by a FTIR spectrometer (dashed green curve), actually excludes the right transitions. and $f_{b}$ and an integer value of $N$ (see the Supplemental Material [24]). In this way, both the lines of Fig. 2 have been univocally identified. The stronger one is a pure rotational line (in fundamental torsional level $\nu_{t}=0$ ) between $(J, K),(19,7)$, and $(20,8)$ rotational levels, while the weaker one also involves a variation of the torsional quantum number $\nu_{t}$, between $\left(J, K, \nu_{t}\right),(12,-9,1)$, and $(12,-8,2)$ levels.

The described procedure gives the coarse calibration of our spectrometer that can now be used for precise measurements of the absolute frequency of the lines identified
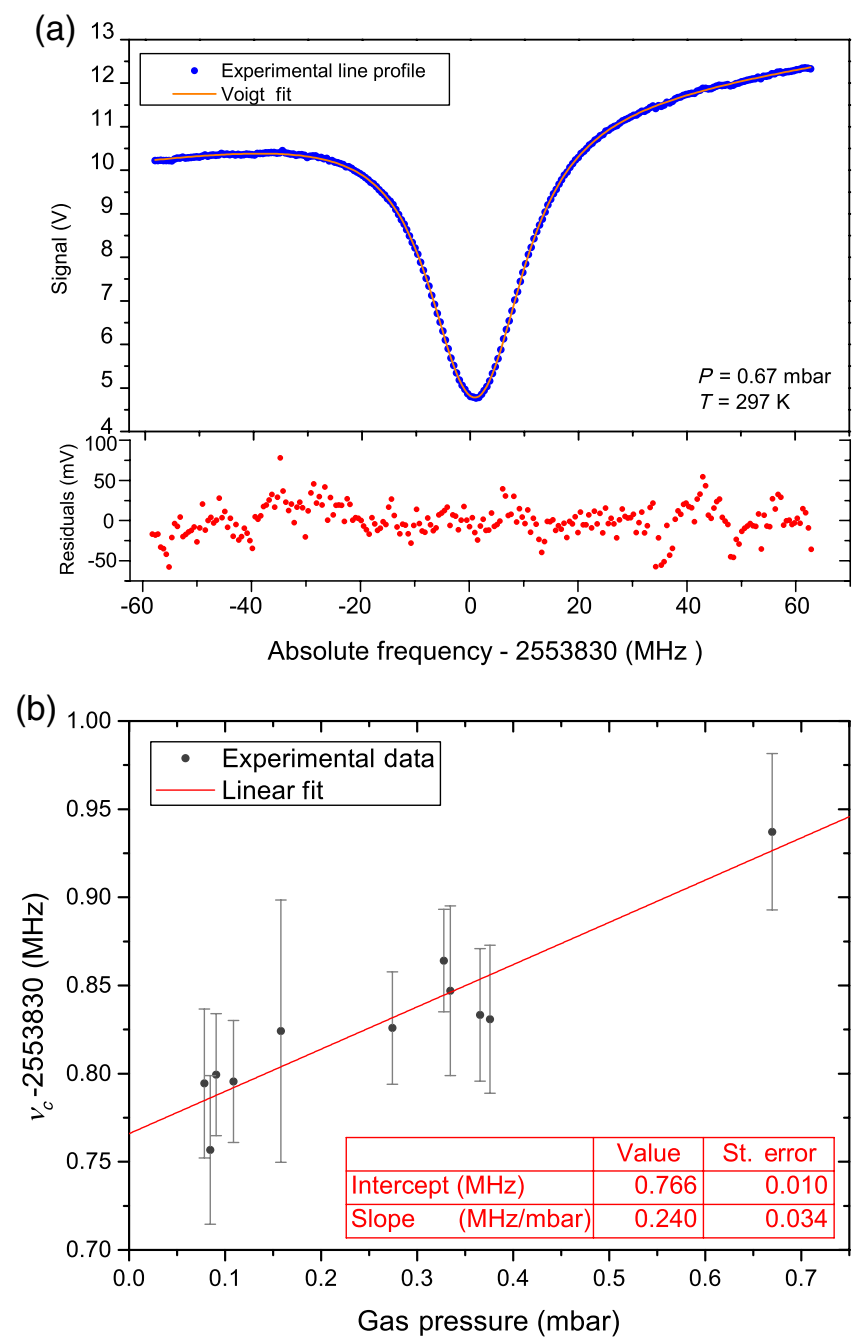

FIG. 4. (a) Experimental absorption profile (blue dots) and Voigt function fit (red line) with residuals (red dots, bottom panel), for the most intense of the two lines investigated. The flat residuals confirm that the adopted fitting function [Voigt profile with linear background; see Eq. (4)] well reproduces the experimental line shape. Gas pressure and temperature during the acquisition are also reported. (b) Dependence of the centerline frequency on gas pressure (black dots), with the corresponding linear fit (red line). The intercept and the slope values of the fit give the absolute frequency $\left(\nu_{c 0}\right)$ and the pressure shift of the considered line. 
above. (In the following, the discussion will be focused on the stronger line.) To this purpose, the line center, as well as other characteristic parameters, are determined by fitting a Voigt function to a set of experimental spectra taken at different pressures [see Fig. 4(a)]. Since, in our case, the line profile is also affected by the dependence of the laser power on the driving current, which is responsible for the linear background visible in the plots of Fig. 4(a), the following fitting function has been used:

$$
\begin{aligned}
S\left(\nu, \nu_{c}, A, w_{G}, w_{L}\right)= & {\left[q+m \cdot\left(\nu-\nu_{c}\right)\right] } \\
& \cdot e^{\left[-\alpha\left(\nu, \nu_{c}, A, w_{G}, w_{L}\right) \cdot L\right]},
\end{aligned}
$$

where $L$ is the cell length, $q$ and $m$ are parameters that describe the linear background, and $\alpha\left(\nu, \nu_{c}, A, w_{G}, w_{L}\right)$ is the Voigt profile describing the absorption coefficient under any condition of temperature and pressure:

$$
\begin{aligned}
\alpha\left(\nu, \nu_{c}, A, w_{G}, w_{L}\right)= & A \frac{2 \ln 2}{\pi^{3 / 2}} \frac{w_{L}}{w_{g}^{2}} \\
& \times \int_{-\infty}^{\infty} \frac{e^{-t^{2}}}{\left(\ln 2 \frac{w_{L}}{w_{G}}\right)^{2}\left(\sqrt{4 \ln 2} \frac{\nu-\nu_{c}}{w_{g}}-1\right)^{2}} d t .
\end{aligned}
$$

Here, $\nu_{c}$ is the line-center frequency, $A$ is the integrated area of the absorption curve, proportional to the transition intensity, while $w_{L}$ and $w_{G}$ are, respectively, the widths (FWHM) of the Lorentzian and Gaussian components of the Voigt profile. The exponential Lambert-Beer law cannot be approximated by the first linear term of its Taylor series, since most of the acquisitions are not in a small absorption regime (absorption larger than 10\% of the total power).

The flat residual plot shown in the bottom panel of Fig. 4(a) confirms the good agreement between the fitting curve and the recorded spectrum, and gives a signal-tonoise ratio (SNR) higher than 200. From the SNR and the linewidth of each Voigt profile (falling in the $\mathrm{MHz}$ range and depending on pressure), the statistical error for the fitted line-center frequency $\left(\nu_{c}\right)$ is retrieved. It ranges from 30 to $40 \mathrm{kHz}$ (depending on the data set), and it is slightly larger than the error given by the fit routine on the $\nu_{c}$ parameter, thus better taking into account other error sources, such as the uncertainty on pressure. The linear dependence on pressure of the line-center frequency is shown in Fig. 4(b). A pressure shift of about $240 \mathrm{kHz} / \mathrm{mbar}$ is measured and, by extrapolating the $\nu_{c}$ value at zero pressure, the line-center absolute frequency is retrieved: $\nu_{c 0}=2553830.766(10) \mathrm{MHz}$.

\section{RESULTS AND DISCUSSION}

The 10-kHz error, given by the linear fit, corresponds to a $4 \times 10^{-9}$ relative uncertainty that is about 2 orders of magnitude worse than the accuracy of the $\mathrm{THz}$ comb. Indeed, we are presently limited by the signal-to-noise ratio of the measurement and by the Doppler-limited spectroscopic resolution. Nevertheless, it is worth making a comparison with the previous measurements on the same line [26,27] and also with the prediction of the most recent molecular models [28] (Fig. 5). In our case, the combination of the absolute referencing provided by the $\mathrm{THz}$ comb with the frequency stability and mW-level power of the QCL allows us to achieve an unprecedented precision. If systematically performed over a large set of lines, measurements at this uncertainty level could help, e.g., to significantly improve theoretical models. Besides the collisional shift that has been observed and studied, other effects such as Zeeman shift, ac-Stark shift, or blackbody shift have been roughly estimated. At the $10-\mathrm{kHz}$ uncertainty level of our measurement, their total contribution is

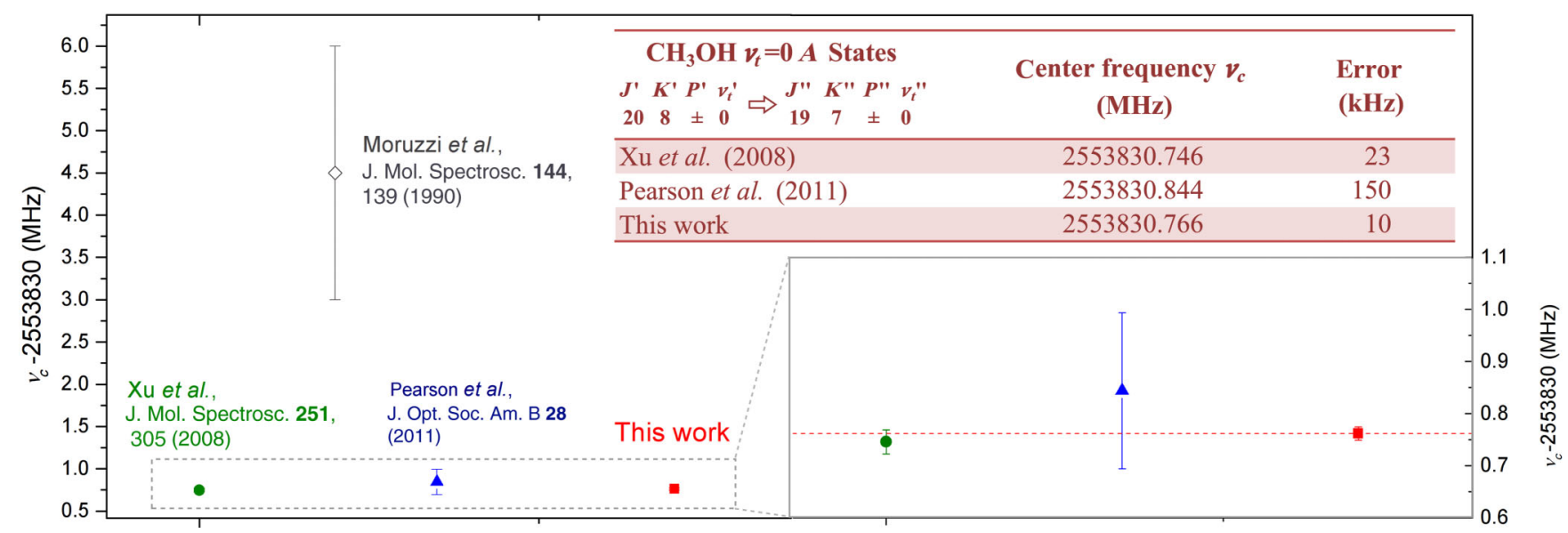

FIG. 5. The absolute frequency of the same transition has been measured by two other techniques: FTIR spectroscopy and microwave spectroscopy. These results (grey and blue, respectively) are compared with ours (red) and with the most recent prediction of a molecular model (green). In particular, the table summarizes the three consistent results zoomed on the right, evidencing the tenfold improvement of the measurement uncertainty provided by our system. 
expected to be below $1 \mathrm{kHz}$, and thus it can be considered negligible. Anyway, in view of future measurements with a much reduced statistical uncertainty, these effects should be carefully taken into account.

The proposed laser-based system provides uncertainties comparable with, or smaller than, microwave-based apparatuses [26] by using, for the spectroscopy, a more compact setup (a 10-cm-long cell instead of a 2.3-m-long one) and a cheap and room-temperature detection (a pyroelectric detector instead of a composite He-cooled Si bolometer). This result is achieved thanks to the significant scaling of about 3 orders of magnitude on the source power (from the $\mu \mathrm{w}$ to the $\mathrm{mW}$ range), which allows us to obtain larger SNRs with shorter optical paths. Moreover, the available power should make it possible to get saturated-absorption signals. The improvement in terms of resolution is expected to be more than 1 order of magnitude, thus giving access to an accuracy at the $10^{-10}-10^{-11}$ level, only limited by the present stability of the comb reference. It is also interesting to note that an improvement of the generation efficiency of the THz comb, as well as of its spectral coverage, will allow us to use a room-temperature detector (Schottky diode, nanowire, or graphene field-effect transistors [29-31]) instead of the hot-electron bolometer for the beat-note detection, thus providing an all-optical, room-temperature setup for referencing metrological-grade $\mathrm{THz}$ sources over a huge spectral window, ranging from the $\mathrm{GHz}$ range up to $5 \mathrm{THz}$ and more. This first demonstration paves the way to new scenarios for a number of different fields, including novel THz-based astronomy [32], high-precision trace-gas sensing [33], and cold-molecule physics [34], and will contribute to filling the still huge $\mathrm{THz}$ gap, in view of upcoming technologies under development in this rapidly evolving spectral window.

\section{ACKNOWLEDGMENTS}

This work was partly supported by the Italian Ministry of Education, University, and Research (MIUR) through the program FIRB-Futuro in Ricerca 2010 RBFR10LULP fundamental research on terahertz photonic devices, by the Extreme Light Infrastructure (ELI) EU roadmap project and by LASERLAB-EUROPE (Grant No. 284464, EC's Seventh Framework Programme). We acknowledge D. Mazzotti and M. Prevedelli for their valuable contribution and discussions.

[1] R. Köhler, A. Tredicucci, F. Beltram, H. E. Beere, E. H. Linfield, A. G. Davies, D. A. Ritchie, R. C. Iotti, and F. Rossi, Terahertz Semiconductor-Heterostructure Laser, Nature (London) 417, 156 (2002).

[2] J. S. Li, W. Chen, and H. Fischer, Quantum Cascade Laser Spectrometry Techniques: A New Trend in Atmospheric Chemistry, Appl. Spectrosc. Rev. 48, 523 (2013).
[3] M. I. Amanti, G. Scalari, M. Beck, and J. Faist, Stand-Alone System for High-Resolution, Real-Time Terahertz Imaging, Opt. Express 20, 2772 (2012).

[4] P. Dean, Y. L. Lim, A. Valavanis, R. Kliese, M. Nikolić, S. P. Khanna, M. Lachab, D. Indjin, Z. Ikonić, P. Harrison, A. D. Rakić, E. H. Linfield, and A. G. Davies, Terahertz Imaging through Self-Mixing in a Quantum Cascade Laser, Opt. Lett. 36, 2587 (2011).

[5] L. Mahler, A. Tredicucci, and M. S. Vitiello, Quantum Cascade Laser: A Compact, Low Cost, Solid-State Source for Plasma Diagnostics, JINST 7, C02018 (2012).

[6] M. Tonouchi, Cutting-Edge Terahertz Technology, Nat. Photonics 1, 97 (2007).

[7] M. S. Vitiello, L. Consolino, S. Bartalini, A. Taschin, A. Tredicucci, M. Inguscio, and P. De Natale, QuantumLimited Frequency Fluctuations in a Terahertz Laser, Nat. Photonics 6, 525 (2012).

[8] M. Ravaro, S. Barbieri, G. Santarelli, V. Jagtap, C. Manquest, C. Sirtori, S. P. Khanna, and E. H. Linfield, Measurement of the Intrinsic Linewidth of Terahertz Quantum Cascade Lasers Using a Near-Infrared Frequency Comb, Opt. Express 20, 25654 (2012).

[9] S. Barbieri, P. Gellie, G. Santarelli, L. Ding, W. Maineult, C. Sirtori, R. Colombelli, H. E. Beere, and D. A. Ritchie, Phase-Locking of a 2.7-THz Quantum Cascade Laser to a Mode-Locked Erbium-Doped Fibre Laser, Nat. Photonics 4, 636 (2010).

[10] M. Ravaro, C. Manquest, C. Sirtori, S. Barbieri, G. Santarelli, K. Blary, J.-F. Lampin, S. P. Khanna, and E. H. Linfield, Phase-Locking of a $2.5 \mathrm{THz}$ Quantum Cascade Laser to a Frequency Comb Using a GaAs Photomixer, Opt. Lett. 36, 3969 (2011).

[11] L. Consolino, A. Taschin, P. Bartolini, S. Bartalini, P. Cancio, A. Tredicucci, H. E. Beere, D. A. Ritchie, R. Torre, M. S. Vitiello, and P. De Natale, Phase-Locking to a FreeSpace Terahertz Comb for Metrological-Grade Terahertz. Lasers, Nat. Commun. 3, 1040 (2012).

[12] K. M. Evenson, D. A. Jennings, and F. R. Petersen, Tunable Far-Infrared Spectroscopy, Appl. Phys. Lett. 44, 576 (1984).

[13] I. G. Nolt, J. V. Radostitz, G. Di Lonardo, K. M. Evenson, D. A. Jennings, K. R. Leopold, M. D. Vanek, L. R. Zink, A. Hinz, and K. V. Chance, Accurate Rotational Constants of $\mathrm{CO}, \mathrm{HCl}$, and HF: Spectral Standards for the 0.3- to 6-THz (10- to 200-cml) Region, J. Mol. Spectrosc. 125, 274 (1987).

[14] L. R. Zink, P. De Natale, F. S. Pavone, M. Prevedelli, K. M. Evenson, and M. Inguscio, Rotational Far Infrared Spectrum of 13CO, J. Mol. Spectrosc. 143, 304 (1990).

[15] M. Bellini, P. De Natale, M. Inguscio, T. D. Varberg, and J. M. Brown, Precise Experimental Test of Models for the Breakdown of the Born-Oppenheimer Separation: The Rotational Spectra of Isotopic Variants of Lithium Hydride, Phys. Rev. A 52, 1954 (1995).

[16] G. Modugno, P. De Natale, M. Bellini, M. Inguscio, G. Di Lonardo, L. Fusina, and J. V. Auwera, Precise Measurement of Molecular Dipole Moments with a Tunable Far-Infrared Stark Spectrometer: Application to $\mathrm{HOCl}$, J. Opt. Soc. Am. B 13, 1645 (1996).

[17] J. C. Pearson, B. J. Drouin, A. Maestrini, I. Mehdi, J. Ward, R. H. Lin, S. Yu, J. J. Gill, B. Thomas, C. Lee, G. 
Chattopadhyay, E. Schlecht, F. W. Maiwald, P. F. Goldsmith, and P. Siegel, Demonstration of a Room Temperature 2.48-2.75 $\mathrm{THz}$ Coherent Spectroscopy Source, Rev. Sci. Instrum. 82, 093105 (2011).

[18] G. Mouret, F. Hindle, A. Cuisset, C. Yang, R. Bocquet, M. Lours, and D. Rovera, $\mathrm{THz}$ Photomixing Synthesizer Based on a Fiber Frequency Comb, Opt. Express 17, 22031 (2009).

[19] F. Hindle, G. Mouret, S. Eliet, M. Guinet, A. Cuisset, R. Bocquet, T. Yasui, and D. Rovera, Widely Tunable $\mathrm{THz}$ Synthesizer, Appl. Phys. B 104, 763 (2011).

[20] T. Yasui, S. Yokoyama, H. Inaba, K. Minoshima, T. Nagatsuma, and T. Araki, Terahertz Frequency Metrology Based on Frequency Comb, IEEE J. Sel. Top. Quantum Electron. 17, 191 (2011).

[21] R. Holzwarth, A. Y. Nevsky, M. Zimmermann, T. Udem, T. W. Hänsch, J. von Zanthier, H. Walther, J. C. Knight, W. J. Wadsworth, P. S. J. Russell, M. N. Skvortsov, and S. N. Bagayev, Absolute Frequency Measurement of Iodine Lines with a Femtosecond Optical Synthesizer, Appl. Phys. B 73, 269 (2001).

[22] L.-S. Ma, M. Zucco, S. Picard, L. Robertsson, and R. S. Windeler, A New Method to Determine the Absolute Mode Number of a Mode-Locked Femtosecond-Laser Comb Used for Absolute Optical Frequency Measurements, IEEE J. Sel. Top. Quantum Electron. 9, 1066 (2003).

[23] S. Yokoyama, R. Nakamura, M. Nose, T. Araki, and T. Yasui, Terahertz Spectrum Analyzer Based on a Terahertz Frequency Comb, Opt. Express 16, 13052 (2008).

[24] See Supplemental Material at http://link.aps.org/ supplemental/10.1103/PhysRevX.4.021006 for details on the search procedure of the matching line, together with the comparison table.

[25] H. M. Pickett, R. L. Poynter, E. A. Cohen, M. L. Delitsky, J.C. Pearson, and H.S.P. Mueller, Submillimeter, Millimeter, and Microwave Spectral Line Catalog, J. Quant. Spectrosc. Radiat. Transfer 60, 883 (1998).
[26] J. C. Pearson, B. J. Drouin, S. Yu, and H. Gupta, Microwave Spectroscopy of Methanol between 2.48 and $2.77 \mathrm{THz}$, J. Opt. Soc. Am. B 28, 2549 (2011).

[27] G. Moruzzi, P. Riminucci, F. Strumia, B. Carli, M. Carlotti, R. M. Lees, I. Mukhopadhyay, J. W. C. Johns, B. P. Winnewisser, and M. Winnewisser, The Spectrum of $\mathrm{CH} 3 \mathrm{OH}$ between 100 and $200 \mathrm{cml}$ : Torsional and Forbidden Transitions, J. Mol. Spectrosc. 144, 139 (1990).

[28] L.-H. Xu, J. Fisher, R. M. Lees, H. Y. Shi, J. T. Hougen, J. C. Pearson, B. J. Drouin, G. A. Blake, and R. Braakman, Torsionrotation Global Analysis of the First Three Torsional States $\left(\nu_{t}=0,1,2\right)$ and Terahertz Database for Methanol, J. Mol. Spectrosc. 251, 305 (2008).

[29] M. S. Vitiello, D. Coquillat, L. Viti, D. Ercolani, F. Teppe, A. Pitanti, F. Beltram, L. Sorba, W. Knap, and A. Tredicucci, Room-Temperature Terahertz. Detectors Based on Semiconductor Nanowire Field-Effect Transistors, Nano Lett. 12, 96 (2012).

[30] M. S. Vitiello, L. Viti, L. Romeo, D. Ercolani, G. Scalari, J. Faist, F. Beltram, L. Sorba, and A. Tredicucci, Semiconductor Nanowires for Highly Sensitive, Room-Temperature Detection of Terahertz Quantum Cascade Laser Emission, Appl. Phys. Lett. 100, 241101 (2012).

[31] L. Vicarelli, M. S. Vitiello, D. Coquillat, A. Lombardo, A. C. Ferrari, W. Knap, M. Polini, V. Pellegrini, and A. Tredicucci, Graphene Field-Effect Transistors as Room-Temperature Terahertz Detectors, Nat. Mater. 11, 865 (2012).

[32] L. Testi, M. Zwaan, C. Vlahakis, and S. Corder, Science Verification Datasets on the ALMA Science Portal, The Messenger 150, 59 (2012).

[33] P. Cancio, S. Bartalini, S. Borri, I. Galli, G. Gagliardi, G. Giusfredi, P. Maddaloni, P. Malara, D. Mazzotti, and P. De Natale, Frequency-Comb-Referenced Mid-IR Sources for Next-Generation Environmental Sensors, Appl. Phys. B 102, 255 (2011).

[34] L. D. Carr, D. DeMille, R. V. Krems, and J. Ye, Cold and Ultracold Molecules: Science, Technology and Applications, New J. Phys. 11, 055049 (2009). 